\title{
Barriers to HIV and sexual and reproductive health care for female sex workers in Tete, Mozambique: results from a cross-sectional survey and focus group discussions
}

Yves Lafort ${ }^{1 *}$, Faustino Lessitala ${ }^{2}$, Balthazar Candrinho ${ }^{3}$, Letitia Greener ${ }^{4}$, Ross Greener ${ }^{4}$, Mags Beksinska ${ }^{4}$, Jenni A. Smit ${ }^{4,5}$, Matthew Chersich ${ }^{1,6}$ and Wim Delva ${ }^{1,7,8}$

\begin{abstract}
Background: In the context of an operational research project in Tete, Mozambique, use of, and barriers to, HIV and sexual and reproductive health (HIV/SRH) commodities and services for female sex workers (FSWs) were assessed as part of a baseline situational analysis.

Methods: In a cross-sectional survey 311 FSWs were recruited using respondent driven sampling and interviewed face-to-face, and three focus group discussions were held with respectively 6 full-time Mozambican, 7 occasional Mozambican and 9 full-time Zimbabwean FSWs, to investigate use of, and barriers to, HIV/SRH care.

Results: The cross-sectional survey showed that $71 \%$ of FSWs used non-barrier contraception, 78 \% sought care for their last sexually transmitted infection episode, 51 \% of HIV-negative FSWs was tested for HIV in the last 6 months, $83 \%$ of HIV-positive FSWs were in HIV care, 55 \% sought help at a health facility for their last unwanted pregnancy and $48 \%$ after sexual assault, and none was ever screened for cervical cancer. Local public health facilities were by far the most common place where care was sought, followed by an NGO-operated clinic targeting FSWs, and places outside the Tete area. In the focus group discussions, FSWs expressed dissatisfaction with the public health services, as a result of being asked for bribes, being badly attended by some care providers, stigmatisation and breaches of confidentiality. The service most lacking was said to be termination of unwanted pregnancies.

Conclusions: The use of most HIV and SRH services is insufficient in this FSW population. The public health sector is the main provider, but access is hampered by several barriers. The reach of a FSW-specific NGO clinic is limited. Access to, and use of, HIV and SRH services should be improved by reducing barriers at public health facilities, broadening the range of services and expanding the reach of the targeted NGO clinic.
\end{abstract}

Keywords: Care seeking, Female sex workers, HIV, Mixed methods, Mozambique, Sexual and reproductive health

\section{Background}

Female sex workers (FSWs) are among the most vulnerable for sexual health risks, as a result of having multiple partners and sexual contacts [1]. The prevalence of HIV and other sexually transmitted infections (STI) among FSWs is several fold the prevalence in the general population [2, 3], HPV infection and resulting cervical cancer

\footnotetext{
*Correspondence: yves.lafort@ugent.be

'International Centre for Reproductive Health, Ghent University, Gent, Belgium

Full list of author information is available at the end of the article
}

are frequent $[4,5]$, unwanted pregnancies are common [6-8], and FSWs are often victims of sexual and other types of violence $[9,10]$. FSWs' access to appropriate health care is hampered by high mobility, possible illegal immigration status, unfamiliarity with, stigmatisation and discrimination at, and inconvenient opening hours of, the local general health services $[11,12]$.

Sex work is common in the adjacent cities of Tete and Moatize in Mozambique. The growing mining industry and the main transport route connecting Malawi to Zimbabwe and the port of Beira attracts travellers, 
migrant labour and sex workers. An enumeration exercise carried out in the Tete-Moatize area in 2008 counted 4,415 FSWs recruiting clients in establishments on a Friday night at the beginning of the month [13]. HIV prevalence among FSWs was $50 \%$ in 2006 [14]. The area (from now on referred to as 'Tete') has 8 public health centres, 1 public hospital, and 4 private clinics, and in Moatize there is a stand-alone drop-in clinic for most-atrisk populations that offers information, education and communication, condoms, STI care, contraceptive services, and HIV testing services (HTS) during the evening (4-10 PM), and is therefore called the Night Clinic. It is operated by a non-governmental organisation, in collaboration with the district health department [13].

Tete is one of the 4 cities participating in the DIFFER project (Diagonal Interventions to Fast-Forward Enhanced Reproductive health), a 5-years operational research project that aims at improving access to HIV/ SRH services for FSWs by a better linkage between interventions targeted at FSWs and the general health services [15]. It applies a methodological framework for health systems research, starting with a situation analysis that informed the development of a context-specific package of interventions to strengthen HIV/SRH service delivery to FSWs. These packages are then implemented and assessed for their feasibility, acceptability, effectiveness, cost-effectiveness and sustainability. The situational analysis was conducted during the first 18 months of the project and applied a convergent parallel mixed methods design, combining multiple qualitative and quantitative research methods $[16,17]$. The objectives were (1) to assess what HIV/SRH services are available at each site and to what extent they are adapted to the needs of FSWs, and (2) to what degree do FSWs make use of the available HIV/SRH services and what are the main barriers. The current article presents the main findings of the second objective of the situational analysis, the use of, and barriers to, HIV/SRH services by female sex workers, in Tete. The findings on the availability and FSW-friendliness of the services are presented elsewhere. The findings from the intervention implementation will be presented at a later stage once the evaluation of the intervention has been completed.

\section{Methods}

The extent of the use of HIV/SRH commodities and services by FSWs was assessed between August 2013 and April 2014 through a cross-sectional survey, using respondent-driven sampling. RDS is similar to snowballing, but corrects for the bias towards FSWs with large social networks through statistical adjustments [18]. RDS begins with the selection of "seeds" who are known members of the FSW population. The seeds are instructed to enrol a limited number of other FSWs from their social circle for the survey, who in turn enrol other FSWs, and so on.

Initially, 6 seeds were recruited of different nationality (Zimbabwean/Mozambican), place of residence (Moatize/ Tete) and number of clients (low/medium/high number of clients) and respondents were requested to each invite 3 new participants using referral coupons. Because of low uptake, the number of seeds was later increased to 13 . Participants were interviewed face-to-face using an electronic questionnaire (QDS ${ }^{\mathrm{mi}}$ Version 2.0) addressing the use of HIV/SRH services and commodities. Coupons were managed using RDS Coupon Manager Version 3.0. Interview data were stored in QDS Data Warehouse and merged with the coupon information. Prevalence estimates and confidence intervals of variables were produced adjusting for unequal probabilities of inclusion due to varying social network sizes and the similarities in characteristics of persons within their social networks, using the RDS analysis package of STATA version 12 (StataCorp). We used the 'Volz-Heckathorn' estimator for the calculation of the weighed proportions and bootstrapping for the confidence intervals, correcting for non-normal distribution [19].

In addition, focus group discussions (FGD) were held with Zimbabwean and Mozambican FSWs for whom sex work was their main occupation ('full-time' FSWs), and Mozambican FSWs for whom it was not ('occasional' FSWs). All Zimbabwean FSWs are full-time FSWs in Tete. Nine FSWs of each type were invited and 9 Zimbabwean, 6 full-time Mozambican and 7 occasional Mozambican FSWs accepted to participate. Using a semi-structured guide in English or Portuguese a moderator, assisted by a note taker, explored access to and experiences with SRH services and recommendations for improving access. The discussions were audio-recorded, transcribed and analysed using NVivo 10 (QSR International) independently by two researchers. The transcripts were thematically inductively coded according to SRH service use, barriers to SRH services, satisfaction with current services, needs for additional services, and recommendations to improve the services. Desire for additional services was explored using a simple preference ranking exercise.

\section{Results}

Cross-sectional survey

Socio-demographic and sex work characteristics (Table 1)

A total of 311 FSWs were recruited. The majority were foreign nationals, $68 \%$ were Zimbabwean, $25 \%$ Mozambican and $7 \%$ of another nationality. The median age of Mozambican FSWs was lower (23.5 years) than of foreign FSWs (30 years). The median number of years living at their current residence was 4 and 2 years (Mozambican and foreign FSWs, respectively). Foreign FSWs had a median number of 15 different partners in the past week, and 
Table 1 Characteristics of surveyed FSWs by nationality $(N=311)$

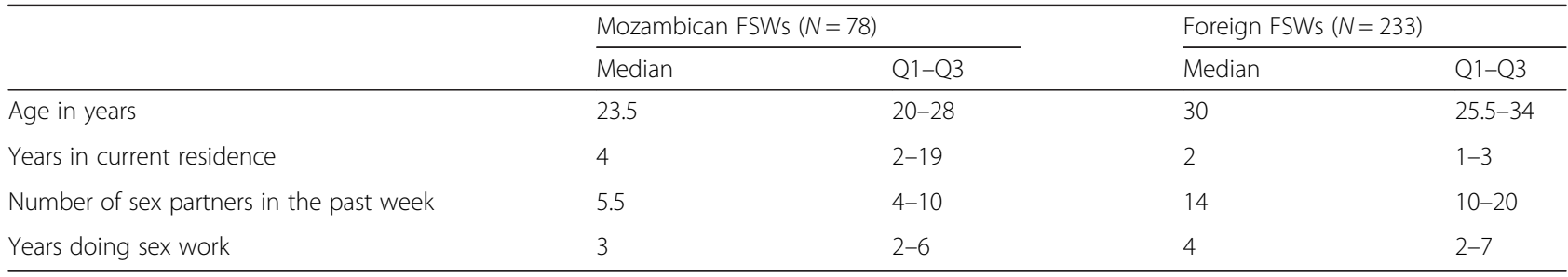

Mozambican FSWs 5. The median number of years engaging in sex work was 3 and 4 years for Mozambican and foreign FSWs, respectively.

\section{Use of HIV and sexual and reproductive health services (Table 2)}

The majority of FSWs (86 \%) who did not want to become pregnant were using a contraception method: $15 \%$ were using condoms only and $71 \%$ a modern nonbarrier contraception method (hormonal, IUD or female sterilisation), of which $64 \%$ combined with condoms (dual protection). When asked what action they had taken, eleven of the 20 FSWs (55\%) who reported an unwanted pregnancy in the past 5 years, replied to have gone to a health facility and the remaining to have done nothing. Approximately half (56 \%) had either an abnormal vaginal discharge or genital ulcers in the past year and $78 \%$ of these $(134 / 172)$ had sought care at a health facility. Half $(51 \%)$ of the FSWs, excluding those who had tested positive for HIV more than 6 months ago, had tested for HIV in the past 6 months. Forty six percent reported to be HIV positive and $83 \%$ of these were enrolled in HIV care and $66 \%$ had already initiated antiretroviral therapy. None of the FSWs had, to their knowledge, ever been screened for cervical cancer. Of the 42 FSWs (16 \%) who had been victim of sexual assault in the past year, about half ( $48 \%$ ) had sought medical care.

Fifteen percent of those who had sought medical care in the past year, reported to have had difficulties in obtaining it, but none reported to have been refused a health service. Half of the FSWs (50 \%) said they disclose that they are a FSW, the majority (96\%) felt they were treated like everybody else and $20 \%$ had feelings of shame or guilt because of being a FSW, when visiting a public health facility.

\section{Place where care sought and satisfaction with received services (Table 3)}

The public health facilities of Tete were the most common source of care for all services, ranging from $38 \%$ for contraceptive services to $78 \%$ for general health care. The Night Clinic was an important provider of contraceptive services (32\%), STI care $(25 \%)$ and to a lesser extent general health care (17\%) and HTS (16 \%). Community outreach was an important source of HTS
(22\%). A third (39\%) of FSWs sought care for HIV and a fifth $(18 \%)$ were last tested for HIV outside Tete.

The most common reasons given for the choice of the place where they last went were that it is where they always go (ranging from $48 \%$ for HTS to $69 \%$ for HIV care), that it is nearby (ranging from $33 \%$ to $44 \%$ ) and that they were referred there (ranging from $17 \%$ to $40 \%$ ). When asked if they were satisfied with the services they had received the last time they sought care, very few FSWs reported to be not or little satisfied, ranging from $0.3 \%$ for general health services to $3.9 \%$ for HIV care.

Care seeking, the place where care is sought, the reasons for the choice and satisfaction with services did not substantially differ between Mozambican and foreign FSWs, or between FSWs who sought care at public health services and the Night Clinic.

\section{Focus group discussions}

The median age of the 9 full-time Zimbabwean FSWs, 6 Mozambican full-time FSWs and 7 Mozambican occasional FSWs participating in the focus group discussions was 36, 23 and 22 years, respectively. The median number of sex partners in the past week was 15, 9.5 and 3, respectively, and the median number of years engaging in sex work 12, 4 and 3.

\section{Need of additional HIV/SRH services}

When asked what services were most in need, Zimbabwean FSWs listed most often termination of pregnancy (TOP). This service was also often listed by occasional Mozambican FSWs and 1 full-time Mozambican FSW. Care for incomplete miscarriages/abortions and services for victims of violence were also often mentioned. Other services listed included more information on certain SRH topics, better STI and HIV care, cervical cancer services, emergency contraception and female condoms.

\section{HIV/SRH care seeking behaviour, barriers and satisfaction}

Mozambican FSWs reported to primarily use the public health facilities for HIV/SRH care. The Night Clinic was known and used by most full time Mozambican FSWs, but most of the occasional FSWs did not know of its existence and one thought it was only intended for Zimbabwean FSWs. Among Zimbabwean FSWs it was known and used by those operating in Moatize. Zimbabwean FSWs reported 
Table 2 Use of HIV and sexual and reproductive health services $(N=311)$

\begin{tabular}{|c|c|c|c|c|}
\hline & $n$ & $\%$ & RDS adjusted \% & $95 \% \mathrm{Cl}$ \\
\hline \multicolumn{5}{|l|}{ Contraception use $(N=253)^{a}$} \\
\hline Used contraception method & 218 & 86.2 & 85.5 & 79.2-91.5 \\
\hline Used condoms only & 38 & 15.0 & 14.1 & $8.9-20.1$ \\
\hline Used non-barrier contraception method & 179 & 70.8 & 70.3 & $62.0-78.1$ \\
\hline \multicolumn{5}{|l|}{ Unwanted pregnancy } \\
\hline Had unwanted pregnancy in the past 5 years & 20 & 6.4 & 7.6 & $2.8-14.1$ \\
\hline Sought help at a health facility $(N=20)^{b}$ & 11 & $55.0 \%$ & - & - \\
\hline \multicolumn{5}{|l|}{ STI care } \\
\hline Had STI symptoms in the past year & 172 & 56.0 & 49.5 & $42.0-57.3$ \\
\hline Sought care at a health facility $(N=172)^{c}$ & 134 & 77.9 & 80.2 & $72.5-87.3$ \\
\hline \multicolumn{5}{|l|}{ HTS $(N=214)^{d}$} \\
\hline Tested for HIV in the last 6 months & 108 & 50.5 & 56.0 & $46.5-65.0$ \\
\hline \multicolumn{5}{|l|}{ HIV care } \\
\hline Reported last HIV test to be positive $(N=278)^{\mathrm{e}}$ & 128 & 46.0 & 46.4 & $38.6-54.4$ \\
\hline Currently in HIV care $(N=128)^{f}$ & 106 & 82.8 & 84.1 & $75.0-25.0$ \\
\hline Currently on ART $(N=128)^{f}$ & 85 & 66.4 & 69.2 & $58.2-80.2$ \\
\hline \multicolumn{5}{|l|}{ Cervical cancer screening } \\
\hline Ever screened for cervical cancer & 0 & 0.0 & - & - \\
\hline \multicolumn{5}{|l|}{ Sexual violence } \\
\hline Victim of sexual violence in past year & 42 & 15.7 & 13.5 & $8.6-19.2$ \\
\hline Sought medical care $(N=42)^{9}$ & 20 & 47.6 & - & - \\
\hline \multicolumn{5}{|l|}{ Attendance at public health facilities } \\
\hline Had difficulty getting health care $(N=121)^{h}$ & 18 & 14.9 & 11.5 & $5.2-19.0$ \\
\hline Was refused a health services in the past 12 months & 0 & 0.0 & - & - \\
\hline Discloses as FSW & 157 & 49.5 & 45.9 & $39.0-53.8$ \\
\hline Feels treated like everybody else & 297 & 96.1 & 94.8 & $91.0-98.3$ \\
\hline Feels shame or guilt because of being FSW & 61 & 19.9 & 19.2 & $13.6-25.4$ \\
\hline
\end{tabular}

${ }^{\mathrm{a}} \mathrm{N}=$ Excludes FSWs with a pregnancy wish or not able to conceive

${ }^{\mathrm{b}} \mathrm{N}=\mathrm{FSW}$ s who had an unwanted pregnancy in the past 5 years

${ }^{c} N=$ FSWs who had STI symptoms in the past year

${ }^{\mathrm{d}} N=$ Excludes FSWs who had tested positive for HIV more than 6 months ago

${ }^{\mathrm{e}} N=$ Excludes FSWs who never tested for HIV

${ }^{\mathrm{f}} \mathrm{N}=$ HIV positive FSWs

${ }^{9} N=$ FSWs who were victim of sexual violence in past year

${ }^{\mathrm{h}} \mathrm{N}=\mathrm{FSW}$ s who sought medical care in the past year

to often use the services of 'bush doctors'. These are illegal providers of modern medicine (oral and injectable medicines), either at the market or at the client's home. Another source of care is the direct purchase of drugs at pharmacies.

The reasons for choosing a public health facility was mostly because it was nearby, but satisfaction with the services was generally very low. The most common reason for dissatisfaction, by all groups of FSWs, was the common practice of asking for a bribe by health providers. If you were willing to pay a bribe, you were rapidly and well attended. But if not, you were either put at the end of the queue, with the risk of not being attended that day, or completely refused the service. This was also the case for services that are supposed to be free and life-saving, such as antiretroviral therapy (ART).

'(translated from Portuguese) ... for me it is the same. Without money I can't get any services. You stay in line and they can send you back home. But with money, all is easy. You can be the first to be attended.' (Mozambican occasional SW)

Another important complaint was the bad reception by staff, especially if they were recognised as a sex worker and/ or refused to pay bribes. This included sometimes refusal 
Table 3 Place where care sought and satisfaction with received services, by type of service

\begin{tabular}{|c|c|c|c|c|c|c|c|c|c|c|}
\hline & \multicolumn{2}{|c|}{ General health care $(N=277)^{\mathrm{b}}$} & \multicolumn{2}{|c|}{ Contraceptive services $(N=213)^{c}$} & \multicolumn{2}{|c|}{ STI care $(N=134)^{d}$} & \multicolumn{2}{|l|}{ HTS $(N=241)^{\mathrm{e}}$} & \multicolumn{2}{|c|}{ HIV care $(N=105)^{f}$} \\
\hline & RDS adjusted \% & $95 \% \mathrm{Cl}$ & RDS adjusted \% & $95 \% \mathrm{Cl}$ & RDS adjusted \% & $95 \% \mathrm{Cl}$ & RDS adjusted \% & $95 \% \mathrm{Cl}$ & RDS adjusted \% & $95 \% \mathrm{Cl}$ \\
\hline \multicolumn{11}{|c|}{ Place where last time care was sought } \\
\hline Public health facilities & 78.0 & $71.7-84.0$ & 37.5 & $29.2-47.1$ & 66.5 & $55.8-75.7$ & 42.8 & $34.3-51.0$ & 60.8 & $48.2-71.9$ \\
\hline Night clinic & 16.8 & $11.7-22.0$ & 30.1 & $21.4-38.8$ & 24.5 & $16.4-34.2$ & 16.1 & $9.6-23.9$ & 0.0 & - \\
\hline Private clinics & 1.8 & $0.5-3.5$ & $(0.8)^{\mathrm{a}}$ & $(0.1-0.5)$ & 0.0 & - & $(0.8)^{\mathrm{a}}$ & $(0.2-3.3)$ & 0.0 & - \\
\hline Informal health sector & 6.6 & $3.4-10.6$ & 15.1 & $9.8-20.9$ & 4.4 & $2.2-8.7$ & 0.0 & - & 0.0 & - \\
\hline Community outreach & 0.0 & - & 4.9 & $2.2-8.3$ & 0.0 & - & 21.9 & $15.8-28.9$ & 0.0 & - \\
\hline Outside Tete & 1.9 & $0.1-4.6$ & 12.8 & 7.9-18.6 & 1.4 & $0.3-4.5$ & 18.0 & $12.5-24.0$ & 39.0 & $27.6-50.1$ \\
\hline \multicolumn{11}{|l|}{ Reason for choice of place } \\
\hline Where I always go & - & - & 65.1 & $55.4-73.9$ & 66.3 & $55.9-75.9$ & 47.8 & $39.1-55.2$ & 69.1 & $58.5-79.2$ \\
\hline Nearby & - & - & 42.5 & $32.9-51.9$ & 43.9 & $32.8-55.3$ & 42.7 & $34.9-50.9$ & 32.6 & $20.9-44.1$ \\
\hline I was referred there & - & - & 17.3 & $11.5-23.4$ & 39.9 & $29.8-51.0$ & 16.4 & $10.3-23.9$ & 19.3 & $11.7-28.5$ \\
\hline Shorter waiting times & - & - & 5.3 & $2.4-9.1$ & 2.8 & $1.6-6.6$ & 9.1 & $4.7-14.4$ & 4.5 & $1.6-10.5$ \\
\hline Privacy & - & - & 3.7 & $1.0-7.6$ & 4.1 & $1.4-8.7$ & 6.3 & $3.1-9.9$ & 10.6 & $4.3-18.3$ \\
\hline Friendly Health personnel & - & - & 1.1 & 6.2 & 0.0 & - & 10.0 & $5.4-15.2$ & 0.0 & - \\
\hline Cost is low or free & - & - & 3.7 & $1.2-7.2$ & $(2.5)^{\mathrm{a}}$ & $(0.0-5.3)$ & 4.4 & $1.7-7.7$ & 2.2 & $0.4-5.0$ \\
\hline Quality of care & - & - & 0.7 & $0.2-2.0$ & 0.0 & - & 2.5 & $0.7-4.9$ & 4.5 & $1.3-10.3$ \\
\hline \multicolumn{11}{|c|}{ Satisfaction with the received services } \\
\hline Very satisfied & 30.0 & $20.1-36.3$ & 29.0 & $20.4-38.6$ & 26.6 & $18.0-36.2$ & 32.4 & $24.4-41.8$ & 33.5 & $22.0-45.4$ \\
\hline Satisfied & 71.7 & $63.3-79.3$ & 70.2 & $61.5-48.8$ & 71.2 & $61.8-80.4$ & 66.9 & $58.3-76.1$ & 62.6 & $51.1-74.7$ \\
\hline Little or not satisfied & 0.3 & $0.3-1.2$ & 0.9 & $0.5-2.8$ & 2.2 & $0.7-5.9$ & $(0.6)^{\mathrm{a}}$ & $(0.1-4.0)$ & 3.9 & $0.5-10.2$ \\
\hline
\end{tabular}

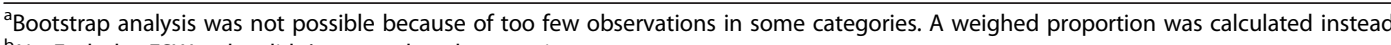

${ }^{\mathrm{b}} N=$ Excludes FSWs who didn't respond to the questions

${ }^{c} N=$ FSWs who used a contraception method and responded to the questions

${ }^{\mathrm{d}} N=$ FSWs who sought care for last STI episode

${ }^{\mathrm{e}} N=$ FSWs who had an HIV test in the past 2 years

${ }^{\mathrm{f}} \mathrm{N}=\mathrm{FSW}$ s who were in HIV care and responded to the question 
to offer services. In particular female providers were said to treat sex workers badly, saying that they were stealing their husbands. The younger occasional Mozambican FSWs reported that they were refused contraceptive services because they were too young. STI care was sometimes refused because of not bringing their partner. It was agreed though that reception by some other providers is very good.

'(translated from Portuguese) ....Me to... I even was beaten, up to been called 'whore, get out of here, or you might seduce our husbands'... (Mozambican full-time FSW)

I usually go to $=X=$ when I have problems. There is one person I do not see anything bad in his service. I always get a good service.' (Zimbabwean FSW)

A third common reason for dissatisfaction, and reason for not using the public services at all, is the lack of privacy and confidentiality. This included fear of being recognised by other users, including their clients and other FSWs, while attending certain services, in particular HIV care; lack of privacy during the consultation; and breaches of confidentiality by the providers. Some FSWs also expressed a concern to be recognised when attending the Night Clinic. Some FSWs do not go for ART out of fear that their partner might discover they are HIV positive.

'For example at $=X=$ hospital, $I$ have sworn never to set my foot there again. Because if I explain my problem to someone, that my vagina is itchy they start telling someone else or another nurse in Portuguese, then start laughing someone else comes to peep at me, I will never see that they are good at their work. In that case ah it is better to go to the Bush Doctors where it will be just the two of us at home.' (Zimbabwean SW)

Other reasons for dissatisfaction were the common drugs shortages, the long waiting times and lengthy lunch breaks, the lack of information and explanations given by providers, and the short consultation time.

Dissatisfaction with the public services was greatest among Zimbabwean FSWs. Because of being foreigners and therefore more easily identifiable as FSWs they are requested to pay higher bribes than Mozambicans and suffer more from bad reception and discrimination. An additional barrier is the language, with few of the health staff speaking Shona or English. Level of dissatisfaction was lowest among occasional FSWs, because they were not recognised as FSW and therefore less discriminated.

...there is this woman hii.., she does not even listen, she does not care because the moment she sees a 'Zimbabweana' [Zimbabwean] she just says puta [prostitute] Oh my God our husbands are gone!...' (Zimbabwean SW)

The FSWs that had used the Night Clinic were highly satisfied with the services mostly because they were well received, the chances to be seen by someone they knew were less, there are fewer clients and the service is fast, and the necessary drugs are always available. Other reasons were that more information is provided, the opening times are convenient, there is no discrimination, the care is better (includes clinical examination and injection) and is free of charge.

I agree with $=X=$, If you go to the private clinic (=Night Clinic=) they have time for you to ask you to lay down and do an examination. If they give you an injection you feel that....yes... you have been treated really.' (Zimbabwean SW)

Zimbabwean FSWs discussed the topic of legalisation of TOP, with most being in favour, but some heavily opposed stating that it would be used as a contraception method. All agreed that post-abortion care needs to be improved and that 'uterus cleaning' should be done. With uterus cleaning they meant evacuation through curettage or aspiration. This service was said to be currently not offered.

\section{Recommendations to improve health services}

When asked what could be done to improve access to services and how they felt about a separate clinic for most-at-risk women, most sex workers were strongly in favour of maintaining the Night Clinic and expanding it to Tete City. Some however were not in favour because of the risk of stigmatisation when you are seen entering the clinic. The consensus was that it was necessary to maintain and expand the Night Clinic, but also improve access to the public services.

\section{Discussion}

We assessed the use of different HIV and sexual and reproductive health commodities and services combining two complementary methods. The cross-sectional survey revealed that the use of HIV and SRH services is often insufficient, with high proportions of FSWs not using a service or commodity they need, but it was not able to identify what the barriers to use are. Few respondents reported difficulties in getting health care and when asked how they feel treated almost all said like everyone else. Very few reported to be dissatisfied with the received services. The only indication of fear of stigmatisation was that half of the FSWs admitted they did not disclose to be a FSW. This is in contrast with the results of the focus group discussions where participants expressed great 
dissatisfaction with the services received at public health facilities.

Being asked for bribes by providers was the most common reason for dissatisfaction, although this is not specific to FSWs but a general practice in the Mozambican public health sector [20]. It is related to factors, such as the poor economic situation of the country, that are beyond the scope of a FSW intervention as ours. Also breaches of confidentiality by the providers is not unique to FSWs. Bad attendance, at least by some providers, was however strongly perceived to be related to being a FSW, as was the fear of being recognised as a FSW by other users. This is consistent with what has been described elsewhere $[11,21,22]$. A better reception and confidentiality can be addressed by sensitising and training providers in FSW-friendly services, but fear of stigmatisation by other users is harder to solve.

Those who had used the Night Clinic clearly preferred it above the public sector. The attendance and the perceived quality of the services is much better, and the fear of stigmatisation is less, although still possible if seen entering the clinic. This is again similar to what was found elsewhere in Africa [11, 23].

Despite being disliked, the public health sector was by far the main provider of HIV/SRH services and the reach of the Night Clinic was limited. The reasons given for choosing the place of care were mostly practical, such as that it is nearby, that it is 'where I always go' or that someone had referred them there. Aspects of quality of care were rarely given as motive. The main reasons why the Night Clinic is still not commonly used are probably that it is located too far from where most FSWs reside and that it is still not widely known, in particular by Mozambican FSWs and FSWs of Tete city.

An important finding was that many FSWs use some HIV/SRH services outside Tete, mostly in their place of origin. The reasons for this are not known. Possible explanations are that FSWs frequently travel to their home area and that they are more acquainted with the services or less stigmatised and discriminated there. This has implications for interventions that aim to improve access to care, in particular HIV care, and it needs to be assessed how linkage with outside services can be improved. The link between mobility and poor retention in HIV care has been well documented but effective strategies to tackle the problem are still lacking $[2,24]$.

The HIV/SRH services offered at the public health facilities and the Night Clinic included contraceptive services, STI care, HTS and HIV care. Services for victims of sexual and gender-based violence did not yet exist; cervical cancer screening was in the process of being introduced and still not widely available; and termination of pregnancy (TOP) was still illegal and only available in exceptional cases at the provincial hospital. Of these services, TOP was the one most mentioned by FSWs in the FGDs as lacking, together with care for postabortion complications. Mozambique has recently legalised TOP and access for FSWs should be ensured as soon as it becomes available.

We combined two complementary methods, each with their strengths and limitations. A cross-sectional survey has the advantage to reach a larger and representative sample of the target population, but does not allow for deeper exploration of the responses given. It is also prone to recollection bias, poor understanding of the question, social desirability bias, or reluctance to divulge sensitive personal information [25]. This probably explains why few FSWs expressed dissatisfaction with the public health services in the cross-sectional survey. An RDS approach facilitates reaching less visible FSWs, but it assumes successful recruitment of participants by their peers. In our study, refusal rate among occasional Mozambican FSWs was high and we believe that they might be underrepresented. Focus group discussions allow a more indepth exploration of the responses and facilitate a more natural discussion, but the representativeness of the participants is not assured and responses can be driven by the more outspoken participants [26]. It is therefore unsurprising that the results of FGDs appear at first sight contradictory with the cross-sectional survey, despite the socio-demographic characteristics of the participants being similar. We argue that rather than viewing these differences a limitation; they confirm the importance of using a mixed-methods approach, yielding complementary results leading to an integrated conclusion [16, 27].

\section{Conclusion}

The use of HIV and SRH services is still insufficient in this female sex worker population. The public health sector is the main provider, but access is hampered by barriers, such as asking for bribes, bad attendance, stigmatisation and breaches of confidentiality. The services at an FSWspecific NGO clinic are more appreciated, but its reach is limited. In a next step, access to, and use of, HIV and SRH services should be improved by reducing the barriers at public health facilities, broadening the range of services offered and expanding the reach by the targeted clinic.

\section{Abbreviations \\ ART, antiretroviral therapy; DIFFER, diagonal interventions to fast-forward expanded reproductive health; FSW, female sex worker; HIV, human immunodeficiency virus; HTS, HIV testing services; IUD, intra-uterine device; NGO, Non-Governmental Organisation; RDS, respondent driven Sampling; $\mathrm{SRH}$, sexual and reproductive health; STI, sexually transmitted infections; TOP, termination of pregnancy}

\section{Acknowledgements}

The authors acknowledge all people who kindly gave their consent to participate in the cross-sectional survey and focus group discussions interviews, as well as the staff of ICRH-Mozambique and the Tete Provincial Department of Health who facilitated the study, and the DIFFER Community and Policy Advisory Board in Mozambique. 


\section{Funding}

The research leading to these results has received funding from the International Department Flanders (DIV) under agreement A11/TT/0382 and the European Union Seventh Framework Programme under grant agreement number Health-F3-2011-282542.

\section{Authors' contributions}

$\mathrm{YL}$ is the coordinator of the DIFFER project and the principal investigator of the situational analysis in Mozambique. He contributed to the development of the study protocol and the data collection tools and adapted them to the Mozambican context. He analysed all the collected information and had the lead in the writing of the survey report and of the article. FL coordinated the data collection in Mozambique, provided feedback to the analysis and contributed to the writing of the survey report and the article. BC was a co-investigator of the situational analysis in Mozambique, provided inputs to the study protocol and data collection tools, and the writing of the survey report and the article. LG is the coordinator of the DIFFER project in South Africa and coordinated the development of the study protocol and data collection tools of the focus group discussions. She also contributed to the writing of the article. RG contributed to the development of the cross-sectional survey protocol and data collection tools, and contributed to the writing of the survey reports and the article. MB provided assistance in the development of the study protocol and data collection tools, and contributed to the writing of the article. JS is the principal investigator of the DIFFER project in South Africa, oversaw the development of the study protocol and data collection tools of the focus group discussions, and contributed to the writing of the article. MC was a principal investigator of the DIFFER project in South Africa and provided inputs to the development of the study protocol and data collection tools, and contributed to the writing of the article. WD Is the PhD promotor of the firs author, provided feedback on the study design, oversaw the analysis of the survey results and the writing of the article. All authors read and approved the final manuscript.

\section{Competing interests}

The authors declare that they have no competing interests.

\section{Ethics approval and consent to participate}

The study protocol was approved by the National Committee of Bioethics for Health in Mozambique (Ref: 358/CNBS/12) and by the Commission for Medical Ethics of the University Hospital Ghent, Belgium (Ref: 2012/843). All participants were thoroughly explained the study procedures and risks, received an explanatory sheet, were invited to participate and, if consenting, signed the consent sheet.

\section{Author details}

${ }^{1}$ International Centre for Reproductive Health, Ghent University, Gent, Belgium. ${ }^{2}$ International Centre for Reproductive Health-Mozambique, Maputo, Mozambique. ${ }^{3}$ Direcção Provincial de Saúde de Tete, Tete, Mozambique. ${ }^{4}$ MatCH Research Unit (Maternal, Adolescent and Child Health Research Unit), Department of Obstetrics and Gynaecology, Faculty of Health Sciences, University of the Witwatersrand, Durban, South Africa. ${ }^{5}$ Discipline of Pharmaceutical Sciences, College of Health Sciences, University of KwaZulu-Natal, Durban, South Africa. ${ }^{6}$ Wits Reproductive Health and HIV Institute, Faculty of Health Sciences, University of the Witwatersrand, Johannesburg, South Africa. ${ }^{7}$ The South African DST/NRF Centre of Excellence in Epidemiological Modelling and Analysis (SACEMA), University of Stellenbosch, Stellenbosch, South Africa. ${ }^{8}$ Center for Statistics, Hasselt University, Diepenbeek, Belgium.

\section{Received: 12 February 2016 Accepted: 14 July 2016}

Published online: 20 July 2016

\section{References}

1. Scorgie F, Chersich MF, Ntaganira I, Gerbase A, Lule F, Lo YR. SocioDemographic Characteristics and Behavioral Risk Factors of Female Sex Workers in Sub-Saharan Africa: A Systematic Review. AIDS Behav. 2012;16(4): 920-33

2. Baral S, Beyrer C, Muessig K, Poteat T, Wirtz AL, Decker MR, et al. Burden of HIV among female sex workers in low-income and middle-income countries: a systematic review and meta-analysis. Lancet Infect Dis. 2012; 12(7):538-49.
3. Pruss-Ustun A, Wolf J, Driscoll T, Degenhardt L, Neira M, Calleja JMG. HIV Due to Female Sex Work: Regional and Global Estimates. Plos One. 2013; 8(5):e63476

4. Cwikel JG, Lazer T, Press F, Lazer S. Sexually transmissible infections among female sex workers: an international review with an emphasis on hard-toaccess populations. Sex Health. 2008;5(1):9-16.

5. Soohoo M, Blas M, Byraiah G, Carcamo C, Brown B. Cervical HPV infection in female sex workers: a global perspective. Open AIDS J. 2013;7:58-66.

6. Morineau G, Neilsen G, Heng S, Phimpachan C, Mustikawati DE. Falling through the cracks: contraceptive needs of female sex workers in Cambodia and Laos. Contraception. 2011;84(2):194-8.

7. Schwartz S, Papworth E, Thiam-Niangoin M, Abo K, Drame F, Diouf D, et al. An Urgent Need for Integration of Family Planning Services Into HIV Care: The High Burden of Unplanned Pregnancy, Termination of Pregnancy, and Limited Contraception Use Among Female Sex Workers in Cote d'Ivoire. J Acquir Immune Defic Syndr. 2015;68:S91-S8.

8. Sutherland EG, Alaii J, Tsui S, Luchters S, Okal J, King'ola N, et al. Contraceptive needs of female sex workers in Kenya - A cross-sectional study. Eur J Contracept Reprod Health Care. 2011;16(3):173-82.

9. Decker MR, Crago AL, Chu SKH, Sherman SG, Seshu MS, Buthelezi K, et al. Human rights violations against sex workers: burden and effect on HIV. Lancet. 2015;385(9963):186-99.

10. Scorgie F, Vasey K, Harper E, Richter M, Nare P, Maseko S, et al. Human rights abuses and collective resilience among sex workers in four African countries: a qualitative study. Glob Health. 2013;9.

11. Scorgie F, Nakato D, Harper E, Richter M, Maseko S, Nare P, et al. 'We are despised in the hospitals': sex workers' experiences of accessing health care in four African countries. Cult Health Sex. 2013;15(4):450-65.

12. Vuylsteke B, Ghys PD, Mah-bi G, Konan Y, Traore M, Wiktor SZ, et al. Where do sex workers go for health care? A community based study in Abidjan, Cote d'Ivoire. Sex Transm Infect. 2001;77(5):351-2.

13. Lafort Y, Geelhoed D, Cumba L, Lazaro CDM, Delva W, Luchters S, et al. Reproductive health services for populations at high risk of HIV: Performance of a night clinic in Tete province, Mozambique. BMC Health Serv Res. 2010;10:144.

14. Lafort $Y$, Sambola F, Joaquim F, Temmerman M, editors. Low prevalence rate of STI among high-risk groups benefitting from improved reproductive health services in Tete province, Mozambique. Mexico City: XVII International AIDS Conference; 2008.

15. ICRH. The DIFFER Project [Available from: http://differproject.eu/. Accessed 15 May 2016.

16. Creswell JW, Plano Clark VL. Designing and Conducting Mixed Methods Research. 2nd ed. Thousand Oaks: Sage Publications; 2011.

17. Curry LA, Krumholz HM, O'Cathain A, Clark VLP, Cherlin E, Bradley EH. Mixed Methods in Biomedical and Health Services Research. Circ Cardiovasc Qual Outcomes. 2013:6(1):119-23.

18. Gile KJ, Handcock MS. Respondent-driven sampling: an assessment of current methodology. In: Liao TF, editor. Sociological Methodology, 2010, vol. 40, no 1. p. 285-327.

19. Salganik MJ, Heckathorn DD. Sampling and estimation in hidden populations using respondent-driven sampling. Sociol Methodol. 2004;34:193-239.

20. USAID. MSI. Mozambique: Corruption Assessment; 2015.

21. Mtetwa S, Busza J, Chidiya S, Mungofa S, Cowan F. "You are wasting our drugs": health service barriers to HIV treatment for sex workers in Zimbabwe. BMC Public Health. 2013:13:7.

22. Ghimire L, Smith WCS, van Teijlingen ER. Utilisation of sexual health services by female sex workers in Nepal. BMC Health Serv Res. 2011;11:8.

23. Stadler J, Delany S. The 'healthy brothel': The context of clinical services for sex workers in Hillbrow, South Africa. Cult Health Sex. 2006:8(5):451-63.

24. Beyrer C, Baral S, Kerrigan D, El-Bassel N, Bekker LG, Celentano DD. Expanding the Space: Inclusion of Most-at-Risk Populations in HIV Prevention, Treatment, and Care Services. J Acquir Immune Defic Syndr. 2011;57:S96-S9.

25. Langhaug LF, Sherr L, Cowan FM. How to improve the validity of sexual behaviour reporting: systematic review of questionnaire delivery modes in developing countries. Tropical Med Int Health. 2010;15(3):362-81.

26. Smithson J. Using and analysing focus groups: Limitations and possibilities. Int J Soc Res Methodol. 2000;3(2):113-9.

27. Wolff B, Knodel J, Sittitrai W. Focus Groups and Surveys as Complementary Research Methods: A Case Example. In: Morgan DL, editor. Successful Focus Groups: Advancing the State of the Art. 1993. 